\title{
Das Verhalten der freien Fettsäuren unter intravenöser Glucosebelastung bei graviden und nicht graviden Patientinnen mit Symptomen eines potentiellen Diabetes mellitus
}

\author{
W.H. Sohilling, L. Herberg, K. Jahnke, K. A. Hüter, und E. Derra
}

2. Medizinische Universitätsklinik und Poliklinik (Direktor: Prof. Dr. K. OberoIssE) und Universitäts-Frauenklinik (Direktor: Prof. Dr. R. EuErr) in Düsseldorf sowie Diabetes-Forschungsinstitut an der Universität Düsseldorf (Leiter: Prof. Dr. K. OBERDISSE)

Eingegangen am 4. April 1968

Response of free fatty acids to an intravenous glucose load in pregnant and non-pregnant women with symptoms of potential diabetes

Summary. In 97 women (aged $18-52$ years) blood glucose and nonesterified fatty acids were determined before and following a rapid intravenous injection of $25 \mathrm{~g}$ glucose $(50 \mathrm{ml}, 50 \%$ solution $) . \quad-49$ of these women were pregnant. For the two main groups (pregnant and nonpregnant), women were selected if they fulfilled one of the following criteria: 1. normal weight without signs of metabolic disorders (control persons); 2. a positive family history of diabetes; 3 . history of unexplained perinatal mortality or previous delivery of an infant weighing 4500 $\mathrm{g}$ or more $; 4$. obesity without signs of metabolic disorders. - We found that the slope of the free fatty acids was re. tarded in pregnant controls. The blood levels of the free fatty acids were always significantly higher than in nonpregnant controls. We even found the same slope of the free fatty acids following an intravenous glucose tolerance test in pregnant women with the above mentioned criteria. In the main group of non-pregnant women with a family history of diabetes or the other diabetic signs, we found a statistically significant difference in the slope of the free fatty acids in 2 of the 3 groups tested compared with controls without metabolic disorders. - These results imply that the raised level of the free fatty acids and the delayed slope following an intravenous glucose tolerance test in healthy pregnant women are due to a lipolytic effect of a placental hormone as already published $[4,23$, 34]. - These findings apply to an elevated lipolysis in healthy pregnant women. Thus pregnancy may have an indirect diabetogenic effect. The reduced lipogenesis, caused by the relative deficiency of insulin in pregnant women with familial history of diabetes, cannot be verified since lipolysis is physiologically raised in pregnancy. During pregnancy the determination of free fatty acids following in intravenous glucose tolerance test is not available for the exact diagnosis of a diabetic metabolism. In non-pregnant women with the symptoms above mentioned, the reduced lipogenesis caused by the relative insufficiency of effective insulin would be verified by the delayed slope of free fatty acids. - Therefore the determination of the concentration of the free fatty acids following an intravenous glucose tolerance test is useful only in non-pregnant women for the diagnosis of latent diabetes.

Changements des taux des acides gras libres après une surcharge glucidique par voie intraveineuse chez des femmes enceintes ou non-enceintes avec des symptômes de diabète potentiel

Résumé. 97 femmes entre 18 et 52 ans ont été soumises à un test de tolérance glucidique par voie intraveineuse avec injection de $25 \mathrm{~g}$ de glucose $(50 \mathrm{ml}$ d'une solution de $50 \%$. Les concentrations du glucose sanguin et des acides gras libres ( $A G L)$ ont été déterminées avant (à jeun) et $10,20,40$ et $60 \mathrm{~min}$ après injection du glucose. -49 des
97 femmes examinées étaient enceintes, tandis que 48 ne l'étaient pas. Chacun des deux groupes était composé d'un groupe de contrôle normal, d'un groupe de femmes avec prédisposition génétique au diabète, d'un autre présentant des symptômes obstétriques et gynécologiques d'un diabète potentiel (naissance d'un enfant mort inexpliqué, naissance d'un ou plusieurs enfants de poids excessif) ot d'un groupe de fermmes obèses. - On constate que la chute des AGL est déjà retardée chez les personnes de contrôle enceintes sans anomalie métabolique apparente. A tous les intervalles examinés ces valeurs sont significativement plus élevées chez ces femmes que chez les personnes de contrôle non-enceintes. La présence de symptômes d'un diabète potentiel n'influence pas la chute des AGL après injection i.v. de glucose, même si on considère le collectif. D'autre part, chez des femmes nonenceintes avec diabète potentiel, nous avons démontré des différences significatives de la chute des AGL dans 2 des 3 groupes examinés par comparaison avec le groupe nor. mal de contrôle. - Ces résultats indiquent que les taux élevés des AGL constatés à tous les intervalles examinés et leur chute retardée après surcharge intraveineuse chez les femmes enceintes normales sont dus à une hormone placentaire avec action lipolytique, telle qu'elle a été décrite dans la littérature $[4,33,34]$. - Il existe done une lipolyse exagérée chez des femmes enceintes sans autre anomalie métabolique. Dans ce sens, une grossesse exerce un effet diabétogène indirect. La présence simultanée d'un diabète potentiel pendant la grossesse ne mène pas à d'autres modifications mesurables de la chute des AGL. - L'augmentation physiologique de la lipolyse ne permet pas de démontrer la diminution de la lipogénèse à laquelle on s'attendrait chez ces femmes à cause du manque relatif d'insuline à effet normal. Pour cette raison, la mesure des taux des AGL sous surcharge glucidique intraveineuse pendant la grossesse n'est pas indiquée pour le dépistage des états prédiabétiques. En dehors de la grossesse et en présence des symptômes indiqués ci-dessus, on peut démontrer un manque relatif d'insuline à action normale par la chute retardée des AGL en comparaison avec le groupe normal de contrôle. En conséquence, les mesures des taux des AGL sous surcharge glucidique intraveineuse pour le dépistage des états prédiabétiques ne sont indiquées qu'en dehors de la grossesse.

Zusammenfassung. Bei 97 Frauen im Alter zwischen 18 und 52 Jahren wurde eine intravenöse Glucosebela. stung mit Injektion von $25 \mathrm{~g}$ Glucose $(50 \mathrm{ml}$ einer $50 \%$ igen Lösung) durchgeführt. Vor (nüchtern) und unter $(10,20$, 40 und 60 Min. nach Injektion der Glucose) der Belastung wurde gleichzeitig die Konzentration des Blutzuckers und der freien Fettsäuren bestimmt. - Von den untersuchten 97 Frauen waren 49 gravide und 48 außerhalb der Schwangerschaft. Diese beiden Gruppen setzten sich jeweils aus einer gesunden Kontrollgruppe, ans einer Gruppe von Frauen mit genetisch diabetischer Belastung, einer anderen mit geburtshilflich-gynäkologischen Symptomen eines 
potentiellen Diabetes (ungeklärte Totgeburt, Geburt eines oder mehrerer Riesenkinder) und aus je einer Gruppe mit fettleibigen Frauen zusammen. - Es zeigt sich, daß der Abfall der freien Fettsäuren schon bei vermutlich stoffwechselgesunden graviden Kontrollpersonen insgesamt verzögert ist. Die einzelnen Werte sind zu allen Bestimmungszeiten bei graviden gesunden Kontrollpersonen statistisch signifikant höher als bei nicht graviden Kontrollpersonen. Das gleichzeitige Vorliegen von Symptomen eines potentiellen Diabetes (genetisch diabetische Belastung, ungeklärte Totgeburten, die Geburt eines oder mehrerer Riesenkinder oder eine Fettleibigkeit) verändert den Abfall der freien Fettsäuren nach intravenöser Glucosegabe auch im Kollektiv nicht. Außerhalb der Gravidität fand sich dagegen bei Patientinnen mit Symptomen eines potentiellen Diabetes in zwei von drei untersuchten Gruppen ein statistisch signifikanter Unterschied im Abfall der freien Fettsäuren im Vergleich zur gesunden Kontrollgruppe. - Diese Befunde lassen sich dahingehend deuten, daß die zu allen Entnahmezeiten erhöht gefundenen Konzentrationen der freien Fettsäuren und der verzögerte Abfall dieser Werte nach intravenöser Glucosebelastung bei gesunden schwangeren Frauen durch ein lipolytisch wirksames Hormon der Placenta bedingt ist, wie das in der Literatur beschrieben wurde $[4,33,34]$. Es besteht somit bei stoffwechselgesunden Graviden eine erhöhte Lipolyse. Eine Schwangerschaft könnte in diesem Sinne indirekt diabetogen wirken. Das gleichzeitige Auftreten von Symptomen eines potentiellen Diabetes bedingt keine weiteren nachweisbaren Veränderungen im Abfall der freien Fettsäuren in der Schwangerschaft. Die schon physiologischerweise erhöhte Lipolyse läßt eine verminderte Lipogenese, die bei solchen Patientinnen infolge relativen Mangels an wirksamern Insulin zu erwarten wäre, nicht mehr nachweisen. Bestimmungen der Konzentration der freien Fettsäuren während intra. venöser Glucosebelastung sind daher innerhalb einer Gravidität für die Erfassung frühdiabetischer Zustände nicht geeignet. - Außerhalb der Gravidität kann demgegenüber bei Vorliegen der oben genannten Symptome ein relativer Mangel wirksamen Insulins aufgrund des verzögerten Abfalls der freien Fettsäuren gegenüber der gesunden Kontrollgruppe vermutet werden. Bestimmungen der Konzentration der freien Fettsäuren nach intravenöser Glucosebelastung sind daher nur außerhalb einer Schwangerschaft zur Erfassung frühdiabetischer Zustände sinnvoll.

Key-words: Free fatty acids, glucose tolerance, pregnancy, potential diabetes, symptoms of potential diabetes.
Mit der Zunahme der Häufigkeit der Zuckerkrankheit in der Gesamtbevölkerung hochzivilisierter Länder haben sich die Bemühungen verstärkt, die diabetische Stoffwechsellage möglichst frühzeitig zu erkennen. In zahlreichen Untersuchungen ließ sich zeigen, daß dem manifesten Stadium des Diabetes mellitus Symptome, die die beginnende Störung des Kohlenhydratstoffwochsels andeuten, vorausgehen können. Dazu gehören die Fettleibigkeit, die Hyperlipidämie und die Neigung zu Spontan-Hypoglykämien. Bei Frauen werden auBerdem die Geburt überschwerer Kinder [1, 25, 26] oder eine Hyperplasie der Langerhans'schen Inseln beim totgeborenen Kind [31] sowie das Auftreten wiederholter Totgeburten [25] als suspekt auf das Vorliegen eines frühdiabetischen Stadiums angesehen. Diese Anzeichen weisen besonders dann auf ein frühdiabetisches Stadium hin, wenn zusätzlich eine familiäre diabetische Belastung besteht. Sie können so als Ausdruck eines potentiellen Diabetes angesehen werden.

HAUSBERgER [23] wies bereits darauf hin, daB dem manifesten Stadium der Zuckerkrankheit häufig eine Störung des Fettstoffwechsels vorausgeht. Eingehende Untersuchungen an familiär diabetisch belasteten Personen bestätigten dieses $[3,10,32,35,47]$. Es fanden sich neben diskreten. Störungen der Glucosetoleranz bei fettleibigen Personen auch erhöhte Insulinwerte im Nüchternblut und eine Erhöhung der Nüchternblutkonzentration freier Fettsäuren. Diese Befunde wurden durch einen relativen Mangel an wirksamem Insulin erklärt. Seit den Untersuchungen von JosLIN [13], Bergquist [2] u.a. $[1,38,48]$ weiß man außerdem, daß die Schwangerschaft einen diabetogenen Effekt hat. Daher sind entsprechende Stoffwechseluntersuchungen bei graviden Patientinnen mit Symptomen eines potentiellen Diabetes mellitus von besonderem Interesse.
Wir haben gravide und nicht gravide Patientinnen, die eines oder mehrere der oben aufgeführten Symptome aufwiesen, intravenös mit Glucose belastet und während dieser Belastung das Verhalten der Konzentration der freien Fettsäuren beobachtet. Damit wollten wir prüfen, ob die Höhe der Nüchternkonzentration und die Art des Abfalls der freien Fettsäuren bei normaler Kohlenhydrattoleranz einen Hinweis auf das Vorliegen eines frühdiabetischen Stadiums geben.

\section{Untersuchungsgut und Methodik}

Insgesamt wurden 97 Frauen untersucht. $49 \mathrm{~Pa}$ tientinnen waren gravide, 48 Patientinnen waren nicht schwanger. Alle graviden Patientinnen wurden in $\mathrm{Zu}$ sammenarbeit mit der Schwangeren-Beratungsstelle der Frauenklinik der Universität Düsseldorf untersucht. Unser Kollektiv der sehwangeren Patientinnen bestand aus 19 Frauen mit genetisch diabetischer Belastung, 7 mit früheren geburtshilflich-gynäkologischen Komplikationen (Geburt eines oder mehrer Riesenkinder, Vorkommen ätiologisch ungeklärter'Totgeburten) und 13 graviden Patientinnen mit einem Ưbergewicht von mehr als $+10 \%(+13 \%$ bis $+118 \%)$ nach Broca. 10 Schwangere haben wir als gesunde Kontrollpersonen untersucht.

Das Kollektiv der nicht graviden Frauen bestand aus zusammen 11 Patientinnen mit genetisch diabetischer Belastung und 12 Frauen, bei denen es während einer früheren Entbindung zu den beschriebenen geburtshilflich-gynäkologischen Komplikationen gekommen war. 10 Patientinnen wurden wegen einer bestehenden Adipositas untersucht und 15 Frauen galten als gesunde Kontrollpersonen. In Tabelle 1 ist das gesamte Kollektiv unter Angabe des Lebensalters, 
der Körpergröße, des Gewichtes, der Schwangerschaftsdauer und des Broca-Indexes wiedergegeben.

Durchführung der intravenösen Glucosebelastung: Alle Untersuchungen wurden morgens bis $9.00 \mathrm{Uhr}$ an den nüchternen Probandinnen durchgeführt. Sie waren an den vorhergehenden Tagen qualitativ und quantitativ ausreichend ernährt worden. Nach Anlegen einer Infusion mit physiologischer Kochsalzlösung hielten die Patientinnen eine Ruhezeit von $30 \mathrm{Min}$. ein. Danach wurden die Nüchternkonzentrationen der freien Fettsäuren und des Blutzuckers bestimmt. AnschlieBend wurden $25 \mathrm{~g}$ Glucose $(50 \mathrm{ml}$ einer $50 \%$ igen Lösung) langsam innerhalb von 2 bis 4 Min. injiziert.
Der Abfall der freien Fettsäuren wurde zusätzlich prozentual zum Ausgangswert berechnet. Dabei galt die Formel:

FFS-Konzentration: $\frac{\text { Nü.-Wert minus 60-Min.-Wert } \times 100}{\text { Nü.-Wert }}$

Die Angabe in den Abbildungen und Tabellen über die Signifikanz entspricht folgenden Werten:

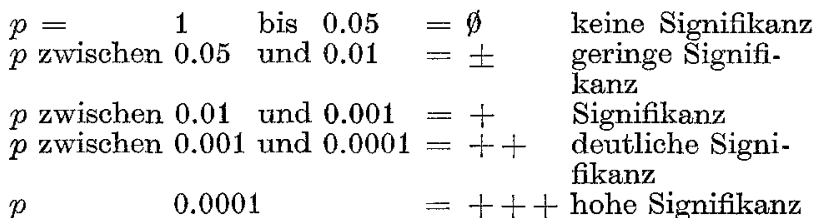

Tabelle 1. Mittelwerte und Angabe des niedrigsten und höchsten Meßwertes für das Lebensalter, die Körpergröße, das Gewicht, Broca-Index und Schwangerschaftsdauer

\begin{tabular}{|c|c|c|c|c|c|c|c|c|c|c|c|c|}
\hline \multirow{2}{*}{\multicolumn{2}{|c|}{$\begin{array}{l}\text { Untersuchte } \\
\text { Gruppe }\end{array}$}} & \multirow{2}{*}{$\begin{array}{l}\text { An- } \\
\text { zahl } \\
n=\end{array}$} & \multicolumn{2}{|c|}{$\begin{array}{l}\text { Alter (Jahre) } \\
\text { im }\end{array}$} & \multicolumn{2}{|c|}{$\begin{array}{l}\text { Größe (ccm) } \\
\text { im }\end{array}$} & \multicolumn{2}{|c|}{$\begin{array}{l}\text { Gewicht (kg) } \\
\text { im }\end{array}$} & \multicolumn{2}{|c|}{$\begin{array}{l}\text { Broca-Index } \\
\text { im }\end{array}$} & \multicolumn{2}{|c|}{$\begin{array}{l}\text { Schwanger } \\
\text { schaftsdauer } \\
\text { (Monate) }\end{array}$} \\
\hline & & & Mitte & I Spanne & Mittel & Spanne & Mittel & Spanne & Mittel & Spanne & Mittel & Spanne \\
\hline \multirow{4}{*}{ 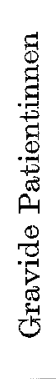 } & $\begin{array}{l}\text { Gesunde } \\
\text { Kontroll- } \\
\text { gruppe }\end{array}$ & 10 & 24.7 & $23-28$ & 163.7 & $\begin{array}{l}157.5- \\
172.0\end{array}$ & 61.0 & $\begin{array}{l}46.0- \\
74.0\end{array}$ & $-13.1 \%$ & $\begin{array}{l}+5 \% \\
-33 \%\end{array}$ & 8.6 & $4-10$ \\
\hline & $\begin{array}{l}\text { Pat. mit } \\
\text { gen.-diab. } \\
\text { Belastung }\end{array}$ & 19 & 27.2 & $18-37$ & 167.7 & $\begin{array}{l}159.0- \\
174.0\end{array}$ & 77.5 & $\begin{array}{l}54.0- \\
111.0\end{array}$ & $+10.0 \%$ & $\begin{array}{l}+69 \% \\
-26 \%\end{array}$ & 8.5 & $6-10$ \\
\hline & $\begin{array}{l}\text { Pat. mit } \\
\text { geb.-gyn. }\end{array}$ & 7 & 29.4 & $23-39$ & 166.7 & $\begin{array}{l}159.0- \\
172.0\end{array}$ & 87.4 & $\begin{array}{r}71.3- \\
126.5\end{array}$ & $+26.7 \%$ & $\begin{array}{l}+5 \%- \\
+83 \%\end{array}$ & 8.5 & $6-10$ \\
\hline & $\begin{array}{l}\text { Pat. mit } \\
\text { Überge- } \\
\text { wicht }\end{array}$ & 13 & 27.5 & $24-36$ & 165.3 & $\begin{array}{l}158.0- \\
174.0\end{array}$ & 91.6 & $\begin{array}{r}71.5- \\
135.0\end{array}$ & $+39.8 \%$ & $\begin{array}{l}+13 \%- \\
+118 \%\end{array}$ & 8.5 & $4-10$ \\
\hline \multirow{4}{*}{ 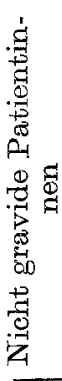 } & $\begin{array}{l}\text { Gesunde } \\
\text { Kontroll- } \\
\text { gruppe }\end{array}$ & 15 & 29.8 & $22-42$ & 169.0 & $\begin{array}{l}159.5- \\
175.0\end{array}$ & 58.1 & $\begin{array}{l}48.0- \\
65.0\end{array}$ & $-2.4 \%$ & $\begin{array}{l}+5 \%- \\
-15 \%\end{array}$ & - & - \\
\hline & Pat. mit & 11 & 34.7 & $25-39$ & 159.5 & $\begin{array}{l}150.0- \\
169.0\end{array}$ & 74.4 & $\begin{array}{r}49.0- \\
117.0\end{array}$ & $+32.7 \%$ & $\begin{array}{l}+89 \% \\
-14 \%\end{array}$ & - & - \\
\hline & $\begin{array}{l}\text { Pat. mit } \\
\text { geb.-gyn. } \\
\text { Anamnese }\end{array}$ & 12 & 38.3 & $30-52$ & 147.8 & $\begin{array}{l}152.0- \\
171.5\end{array}$ & 68.8 & $\begin{array}{l}53.0- \\
90.0\end{array}$ & $+18.7 \%$ & $\begin{array}{l}+7 \%- \\
+48 \%\end{array}$ & - & - \\
\hline & $\begin{array}{l}\text { Pat. mit } \\
\text { Ubuer- } \\
\text { gewicht }\end{array}$ & 10 & 35.6 & $28-42$ & 158.6 & $\begin{array}{l}152.0- \\
167.0\end{array}$ & 66.5 & $\begin{array}{l}61.5- \\
73.0\end{array}$ & $+25.7 \%$ & $\begin{array}{l}+12 \%- \\
+47 \%\end{array}$ & - & - \\
\hline \multicolumn{2}{|c|}{ Insgesamt } & 97 & 30.9 & $18-52$ & 162.3 & $\begin{array}{l}150.0- \\
175.0\end{array}$ & 73.2 & $\begin{array}{r}46.0 \\
135.0\end{array}$ & $+17.3 \%$ & $\begin{array}{l}-33 \%- \\
+118 \%\end{array}$ & 8.5 & $4-10$ \\
\hline
\end{tabular}

Weitere Bestimmungen der Blutzucker- und freien Fettsäurekonzentration erfolgten 10, 20,40 und 60 Min. nach Beendigung der Injektion.

Die freien Fettsäuren wurden nach der Methode von Dole $[15,16]$, modifiziert nach LocHNER u. Mitarb. [37] bestimmt. Die Bestimmung der Blutzuckerkonzentration erfolgte mittels Autoanalyzer Technicon mit der auf den Autoanalyzer adaptierten RedoxReaktion nach GRADY u. LAMAR [22], modifiziert nach Hofwinand [27]. Die Glucosetoleranz bewerteten wir nach rechnerischer Ermittlung der Konstanten, wie sie CONARD [12] angegeben hat. Sie ergibt sich aus der Gleichung:

$$
K \times 10^{2}=\frac{\log 10 C_{1}-\log 10 O_{2}}{t_{2}-t_{1}} \cdot \log n 10 \times 100
$$

Die Beurteilung der Glucosetoleranz erfolgte nach den an anderer Stelle von uns veröffentlichten Kriterien [42]. Die Konstante nach Conard galt als pathologisch, wenn der Wert $K \times 10^{2}$ kleiner als 1.40 war. Werte von 1.40 und darüber galten als normal.

Der Abfall der Konzentration der freien Fettsäuren unter intravenöser Glucosebelastung wurde als normal angesehen, wenn weniger als drei von fünf Meßwerten unter einer aus dem jeweiligen Kontrollkollektiv ermittelten oberen Normalgrenze lagen.

Die Ermittlung des Sollgewichtes erfolgte nach der Broca'schen Formel. Dabei galt für Frauen folgende Beziehung:

Körpergröße in Zentimetern minus 100 minus $10 \%$ 
Die Subtraktion eines Wertes von $10 \%$ ist wegen der besonderen Konstitution und des leichteren Knochenbaues bei Frauen erforderlich.

\section{Ergebnisse}

A) Verhalten der freien Fettsäuren (FFS) über 60 Min. nach Injektion von $25 \mathrm{~g}$ Glucose bei gesunden nicht graviden und gesunden graviden Frauen.

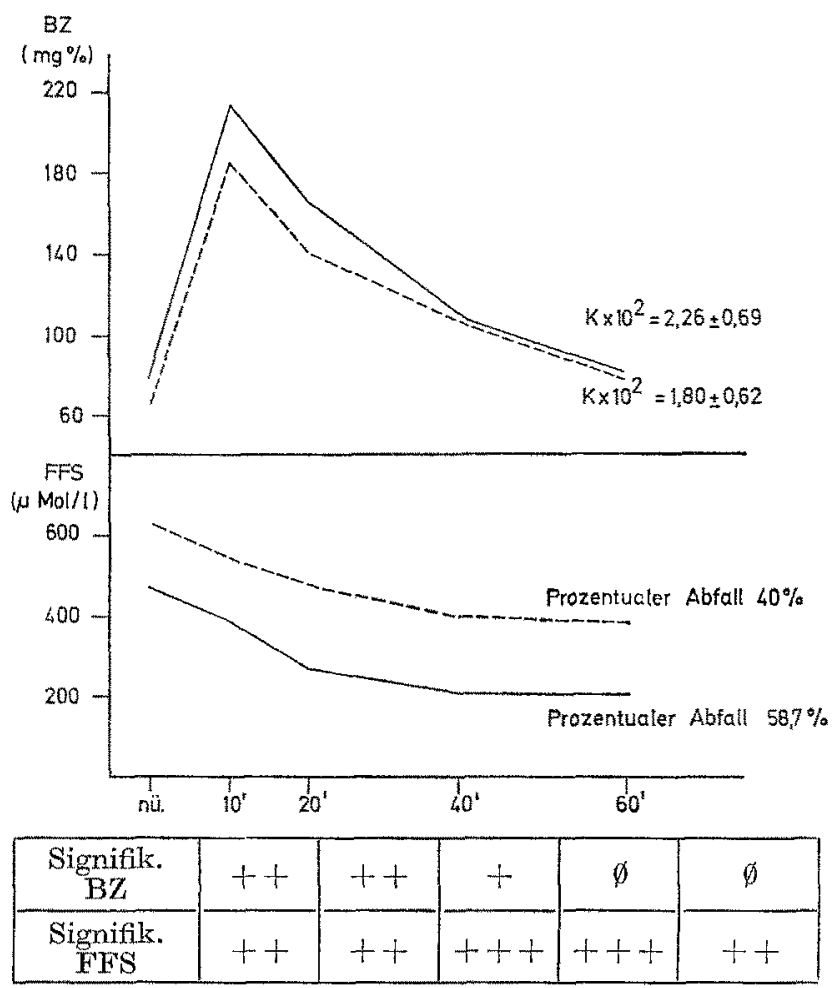

Abb. 1. FFS und $B Z$ bei gesunden nicht-graviden und gesunden graviden Kontrollpersonen unter Angabe der Sig* nifikanz des Unterschieds

Gravide Kontrollpersonen $(n=10)$

Nicht gravide Kontrollpersonen $(n=15)$

Insgesamt konnten 15 Frauen (Kontrollkollektiv) zwischen 22 und 42 Jahren untersucht werden. Alle Patientinnen waren normgewichtig und gaben anamnestisch und klinisch keine Hinweise auf das Vorliegen eines potentiellen Diabetes mellitus. Wie aus Abb. $1 \mathrm{zu}$ ersehen ist, kommt es bei nicht graviden, vermutlich stoffwechselgesunden Fraven nach intravenöser Glucosegabe zu einem deutlichen Abfall der freien Fettsäurekonzentration. Dieser Abfall setzt schon in der Phase der noch ansteigenden Blutzuekerkonzentration, also bis zur 10. Min. post injectionem, verstärkt ein. Während der Blutzuckerspiegel anschließend in Form einer Hyperbel abfällt $\left(K \times 10^{2}=2.26 \pm 0.69\right)$, kommt es zu einem weiteren ständigen Abfall der freien Fettsäurekonzentration bis zur 60. Min. nach der Injektion. Der Wert für den prozentualen Abfall dex freien Fettsäuren liegt bei 58.7 .
Vergleicht man diese Werte (Abb. 1) mit den entsprechenden der 10 gesunden graviden Kontrollper. sonen, die im zweiten und dritten Trimester der Schwangerschaft (Alter zwischen 23-28 Jahren) in gleicher Weise untersucht wurden, so ergibt sich folgendes Bild: Die Konzentration der FFS gravider Frauen ist zu allen Entnahmezeiten gegenüber derjenigen nicht gravider Kontrollpersonen deutlich und signifikant erhöht. Der Abfall freier Fettsäuren gravider Frauen ist besonders initial verzögert. Sein prozentual berechneter Wert beträgt 40. Demgegenüber zeigt die Blutzuckerkonzentrationskurve niedrigere Werte als in der Gruppe der nicht graviden gesunden Kontrollpersonen. Auch der Nüchtern-Blutzucker liegt in der Gravidität ( $67 \pm 6 \mathrm{mg} \%$ ) niedriger als außerhalb der Schwangerschaft $(76 \pm 11 \mathrm{mg} \%)$. Die Konstante nach Conard liegt in der Schwangerschaft mit $1.80 \pm$ 0.62 niedriger als außerhalb der Gravidität $(2.26 \pm$ 0.69). Aufgrund dieser Befunde ist zu vermuten, daß schon durch eine normale Gravidität der Fettstoffwechsel beeinflußt wird, ohne daf Hinweise auf das Bestehen eines potentiellen Diabetes mellitus vorhanden sind.

B) Verhalten der freien Fettsäuren (FFS) über 60 Min. nach Injektion von $25 \mathrm{~g}$ Glucose bei Patientinnen mit Symptomen eines poteniiellen Diabetes mellitus innerhalb der Schwangerschaft.

In dieser Gruppe haben wir 19 Frawen mit genetisch diabetischer Belastung, 7 wegen früher aufgetretener gynäkologisch-geburtshilflicher Komplikationen und 13 fettleibige Patientinnen untersucht.

Die Abb. 2 zeigt das Verhalten der FFS nach Injektion von $25 \mathrm{~g}$ Glucose im Vergleich zur gesunden Kontrollgruppe. Es ist zu ersehen, daß sich keines der drei untersuchten Kollektive von der gesunden Kontrollgruppe wesentlich unterscheidet. Es ergibt sich weder hinsichtlich des Abfalls der Blutzuckerkonzentration noch der Konstanten nach Conard noch des Abfalls der FFS ein im Vergleich zur gesunden Kontrollgruppe wesentlicher Unterschied.

Die Befunde lassen vermuten, daß der schon bei gesunden graviden Kontrollpersonen beobachtete initial verzögerte Abfall der FFS, der auch zu späteren Zeiträumen auf einem vergleichsweise höheren Niveau erfolgt, durch das gleichzeitige Vorliegen von Sym. ptomen eines frühdiabetischen Stadiums nicht weiter verändert werden kann. Bestimmungen der FFS in der Schwangerschaft unter intravenöser Glucosebelastung ergeben demnach keinen Hinweis auf eine beginnende Š̈örung des Kohlenhydratstoffwechsels im Sinne eines frühdiabetischen Stadiums.

C) Verhallen der freien Fettsäuren (FES) über 60 Min. nach Injeltion von $25 \mathrm{~g}$ Glucose bei Patientinnen mit Symptomen eines potentiellen Diabetes mellitus außerhalb der Schwangerschaft.

Insgesamt konnten in dieser Gruppe 11 Frauen mit genetisch diabetischer Belastung, 12 Patientinnen mit 
früheren geburtshilflich-gynäkologischen Komplikationen und 10 fettleibige Frauen untersucht werden.

Die Ergebnisse unserer Untersuchungen sind in Abb. 3 zusammenfassend dargestellt. Gegenüber der gesunden Kontrollgruppe läßt sich bei der Gruppe der genetisch diabetisch belasteten Personen eine stati- stisch signifikant erniedrigte Konstante nach Conard nachweisen. Auch der Abfall der FFS unterscheidet sich gegenüber dem der Kontrollgruppe zum Teil signifikant. Bei erhöhtem Nüchternwert ist ein deutlich verzögerter Abfall der FFS nach Glucosegabe zu beobachten. Der prozentuale Abfall liegt hier bei 51 gegen.

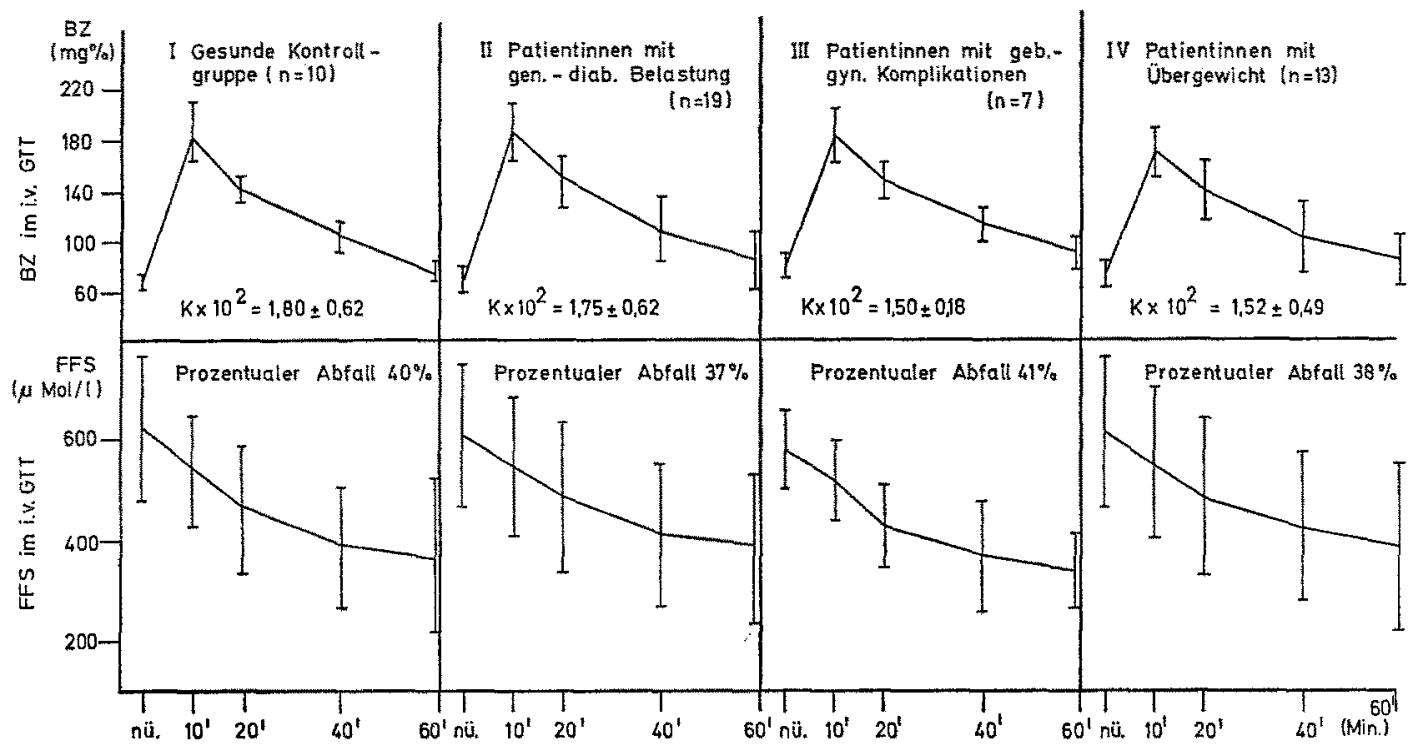

\begin{tabular}{|ll|l|l|l|l|l|l|l|l|l|l|l|l|l|l|l|}
\hline $\begin{array}{l}\text { Signifikanz zur } \\
\text { N.Gruppe }\end{array}$ & BZ & $\emptyset$ & $\emptyset$ & $\emptyset$ & $\emptyset$ & $\emptyset$ & ++ & $\emptyset$ & $\emptyset$ & $\emptyset$ & ++ & $\emptyset$ & $\emptyset$ & $\emptyset$ & $\emptyset$ \\
\hline FFS & $\emptyset$ & $\emptyset$ & $\emptyset$ & $\emptyset$ & $\emptyset$ & $\emptyset$ & $\emptyset$ & $\emptyset$ & $\emptyset$ & $\emptyset$ & $\emptyset$ & $\emptyset$ & $\emptyset$ & $\emptyset$ \\
\hline
\end{tabular}

$\mathrm{Abb}$. 2. Verhalten der Glucosekonzentration und dex Konzentration der FFS unter i.v. Glucosebelastung in den untersuchten Kollektiven innerhalb der Schwangerschaft $\left(\bar{x} \pm 1 S_{D}\right)$

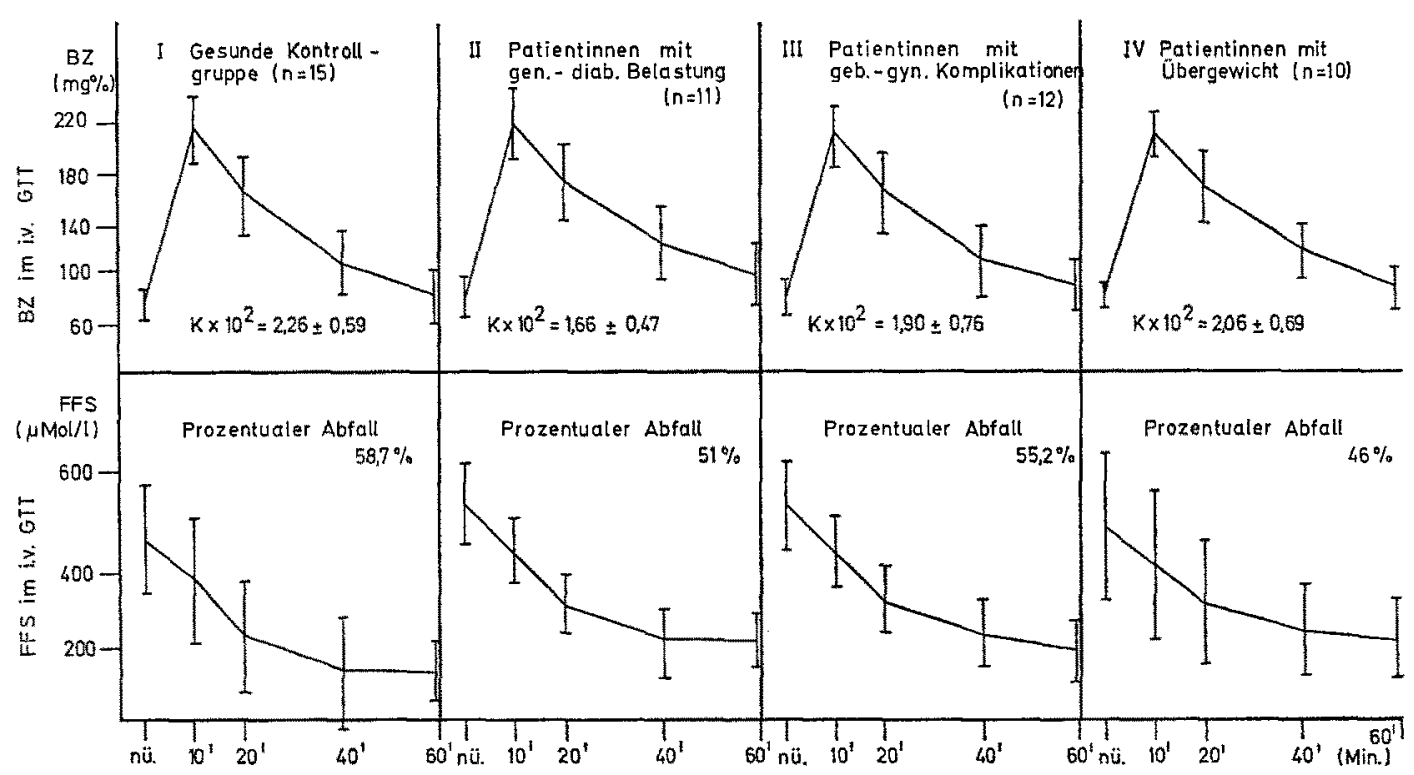

\begin{tabular}{|ll|l|l|l|l|l|l|l|l|l|l|l|l|l|l|l|}
\hline $\begin{array}{l}\text { Signifikanz zur } \\
\text { N-Gruppe }\end{array}$ & FES & \pm & $\emptyset$ & + & \pm & $\emptyset$ & + & $\emptyset$ & + & + & $\emptyset$ & $\emptyset$ & $\emptyset$ & $\emptyset$ & + & $\emptyset$ \\
\hline
\end{tabular}

Abb. 3. Verhalten der Blutzuckerkonzentration und der Konzentration der FFS unter i.v. Glucosebelastung in den untersuchten Kollektiven außerhalb der Schwangerschaft 
über 58,7 in der Kontrollgruppe. Auch bei den Patientinnen, die anamnestisch die Geburt eines Riesenkindes oder das Auftreten einer ätiologisch ungeklärten Totgeburt angaben, findet sich ein gegenüber dem Kontrollkollektiv unterschiedlicher Abfall der FFS-Konzentration. Nüchtern-Wert, 20- und 40-Min.-Wert unterscheiden sich statistisch signifikant gegenüber den entsprechenden Werten der gesunden Kontrollgruppe. Demgegenüber läßt sich ein Unterschied im Abfall der Blutzuckerkonzentration und der Konstanten nach Conard nicht nachweisen. In der untersuchten Gruppe von 10 fettleibigen Frauen mit einem Übergewicht von durchschnittlich $+25.7 \%$ nach Broca $(+12 \%$ bis $+47 \%$ ) konnten keine statistisch signifikanten Unterschiede im Vergleich zur gesunden Kontrollgruppe nachgewiesen werden, allerdings liegt der prozentuale Abfall um 12 niedriger als in der Kontrollgruppe. Auch der Abfall der Blutzuckerkonzentration und die Konstante nach Conard unterscheiden sich in dieser Gruppe nicht wesentlich rom gesunden Kontrollkollektiv.

Zusammenfassend läßt sich sagen, daß der Abfall der FFS unter intravenöser Glucosbelastung bei nicht graviden Frauen mit anamnestischen Hinweisen auf das Bestehen frühdiabetischer Stoffwechselveränderungen gegenüber einem entsprechenden vermutlich stoffwechselgesunden Kontrollkollektiv auf einem höheren Niveau eher verzögert abläuft. Es muß allerdings betont werden, daß von den 33 Frauen nur 5 normgewichtig waren. 27 Frauen hatten ein Übergewicht von $+10 \%$ und mehr nach Broca, so daß die Veränderungen im Fett- und Kohlenhydratstoffwechsel möglicherweise auf das Vorhandensein der Adipositas zurückzuführen sind. Die untersuchten Kollektive erlauben es jedenfalls nicht, auf eine Wertigkeit der einzelnen Symptome hinsichtlich des Vorliegens eines frühdiabetischen Zustandsbildes zu schließen.

Trotz der relativ geringen Zahl der in den einzelnen Kollektiven untersuchten Personen deuten die vorgelegten Ergebnisse darauf hin, daß ein Unterschied besteht in bezug auf den Abfall der freien Fettsäuren bei vermutlich stoffwechselgesunden graviden Frauen und gesunden nicht graviden Frauen. Symptome eines potentiellen Diabetes in der Schwangerschaft zeigen keinen weiteren Einfluß auf den Abfall der freien Fettsäuren.

Außerhalb der Gravidität läßt sich bei Patientinnen mit Symptomen eines potentiellen Diabetes eine gewisse Veränderung im Abfall der freien Fettsäuren unter intravenöser Glucosebelastung beobachten, wie das in der Literatur beschrieben ist.

\section{Diskussion}

Die dargelegten Befunde weisen auf eine Beeinflussung des Intermediärstoffwechsels durch die Schwangerschaft bei stoffwechselgesunden Personen hin. Die Konzentration der freien Fettsäuren gesunder gravider Frauen ist gegenüber derjenigen nicht gravider Frauen zu allen Zeiten der Blutentnahme unter intravenöser Glucosebelastung deutlich und signifikant erhöht.

Der gleiche Befund ist bereits aus der Literatur bekannt $[6,7,8,11,13,39,43,44]$. Von den meisten Autoren wird diese Beobachtung durch eine in der Schwangerschaft herabgesetzte periphere Glucoseutilisation im Sinne eines primär diabetogenen Effektes der bestehenden Gravidität gedeutet. Einer solchen Annahme würde auch die Beobachtung einer herabgesetzten Kohlenhydrattoleranz nach oraler Glucosebelastung und nach oraler Cortison-Glucose-Belastung entsprechen $[28,29,30]$. Demgegenüber ließ sich jedoch in Untersuchungen von HÜTER u. Mitarb. [29] bei der intravenösen Glucosebelastung keine herabgesetzte Kohlenhydrattoleranz nachweisen. Die Konstante nach Conard zeigte einen unteren Normwert von 1.46 (Mittelwert 1.92 minus doppelter Standardabweichung, also minus 0.46). Zu gleichen Ergebnissen kam auch Freinkel [18]. Die scheinbar verminderte Glucosetoleranz nach oraler einfacher Glucosebelastung oder kombinierter Cortison-Glucose-Belastung wird durch die veränderten Resorptionsverhältnisse und durch die Vermehrung der interstitiellen Flüssigkeit erklärt.

Die Annahme einer primär verschlechterten Kohlenhydrattoleranz im Sinne einer frühdiabetischen Veränderung erscheint daher nicht gesichert. Dementsprechend ist es auch fraglich, ob die Erhöhung der Konzentration der freien Fettsäuren bei gesunden graviden Frauen nüchtern und nach Glucoseinjektion als Ausdruck eines primär diabetogenen Effektes der Schwangerschaft und somit eines relativen Mangels wirksamen Insulins und dadurch verminderter Lipogenese angesehen werden kann.

Bestimmungen der Seruminsulinkonzentrationen bei gesunden graviden Frauen unter oraler und intravenöser Glucosebelastung sprechen ebenfalls nicht eindeutig für einen primär diabetogenen Effekt der Gravidität. Hohe Insulinspiegel nach i.v.-Glucosebelastung wurden von Speclacy u. Goetz [45, 46] und von Bletcher u. Mitarb. [5] beobachtet. Lambert $u$. Mitarb. [36], Crostgnant [14] und Gestaldi [20] fanden demgegenüber bei immunologischer Bestimmung des Insulins unter oraler Glucosebelastung keinen Unterschied zwischen gesunden graviden und gesunden nicht graviden Frauen. Diese widersprüchlichen Befunde sind möglicherweise durch unterschiedliche Untersuchungs- und Bestimmungsverfahren zu erklären. In keinem Fall geben sie Hinweise für einen primär diabetogenen Effekt der gesunden Gravidität, die zu einer Erhöhung der Konzentration der freien Fettsäuren infolge herabgesetzter Lipogenese führen würde.

Nach neueren Untersuchungen dürften die erhöhten Konzentrationen der freien Fettsäuren weniger durch eine herabgesetzte Lipogenese als vielmehr durch eine gesteigerte Lipolyse bedingt sein. So ist seit den Untersuchungen von ELERT [17] bekannt, daß es während der Gravidität zu einem ,,adaptiven Hypercorticismus" kommt. Infolge des parasitenähnlichen Wachs- 
tums des Föten soll es nach den Untersuchungen von RoTH [41] zu einem relativen Hungerzustand der Mutter und so zur vermehrten Ausschüttung der lipo. lytisch wirksamen Hormone Glucagon und Somatotropin kommen. Josimovich u. MoLaREN [33] und andere Autoren [4, 34] konnten außerdem aus der menschlichen Placenta eine Substanz isolieren, die sich immunologisch wie das Wachstumshormon verhält und als ein schwangerschaftsspezifisches Hormon angesehen wird. Unter Berücksichtigung dieser Befunde und der von RandLE u. Mitarb. [19, 40] beschriebenen herabgesetzten Empfindlichkeit des Muskelgewebes gegenüber Plasmainsulin bei erhöhter Konzentration der freien Fettsäuren ist es verständlich, daß die Gravidität nur als sekundärer diabetogener Faktor anzusehen ist. Bei prämorbiden Patientinnen kann auf diesem Wege die Schwangerschaft zur Manifestation eines Diabetes mellitus führen. Es wäre daher auch zu vermuten, daß bei graviden Frauen mit den oben beschriebenen Symptomen eines potentiellen Diabetes eine besondere Gefährdung des Stoffwechsels vorliegt, die unter entsprechender Belastung biochemisch im Verhalten der Blutzuckerkonzentration und der freien Fettsäurespiegel nach intravenöser Glucosebelastung nachweisbar sein sollte.

In unseren Untersuchungen konnte diese Annahme nicht bestätigt werden. Man kann die Befunde nur dahingehend deuten, daß die physiologischerweise gesteigerte Lipolyse in der Schwangerschaft eine verminderte Lipogenese infolge kurzfristigen relativen Mangels an wirksamem Insulin nach intravenöser Glucosebelastung nicht mehr nachweisen läßt. Das bedeutet, daß frühdiabetische Veränderungen, wie sie gerade bei Personen mit Symptomen eines potentiellen Diabetes mellitus gehäuft vorkommen, durch ein abnormes Verhalten im Abfall der freien Fettsäuren nach intravenöser Glucosegabe in der Gravidität nicht nachweisbar sind. Messungen der freien Fettsäuren im Serum solcher Patientinnen nach intravenöser Glucosebelastung sind daher bei graviden Frauen zur Erfassung eines frühdiabetischen Stadiums ohne diagnostischen Wert.

Die Situation außerhalb der Schwangerschaft scheint anders zu sein. Hier besteht ein Gleichgewicht zwischen Lipogenese und Lipolyse [9, 21, 24]. Eine Ausschüttung wirksamen Insulins aus den Betazellen des Inselorgans, wie sie nach intravenöser Glucosebelastung zu beobachten ist, kann zu einer gesteigerten Lipogenese und dadurch zu einem meßbaren Abfall der freien Fettsäuren unter Glucoseeinwirkung führen. Bei Patientinnen mit Symptomen einer frühdiabetischen Stoffwechselstörung ist ein Mangel wirksamen Insulins zu vermuten, so daß solche Kollektive im Mittel gegenüber entsprechenden Kontrollkollektiven einen verzögerten Abfall der freien. Fettsäuren auf höherem Niveau zeigen. Man darf daraus schließen, daß die Erfassung des Konzentrationsabfalls der freien Fettsäuren nach intravenöser Glucosegabe außerhalb der Gravidität beim Vorliegen bestimmter Symptome, die auf ein frühdiabetisches Stadium hinweisen, einen gewissen diagnostischen Wert hat. Während der Gravidität ist dieses nicht der Fall.

Für die technische Hilfe bei der Durchführung dieser Untersuchungen sind wir Frau L. MIss, FrI. K. BoHMERT, Frl. Ch. Hesse-Wortmany und Frl. B. Fuermanns zu Dank verpflichtet.

\section{Literatur}

1. BEEK, v. C.: Embryopathia diabetica und die Diagnostik des mütterlichen Diabetes mittels Sektion ihres totgeborenen Kindes. 3. Symposion Dtsch. Ges. Endokrinologie Bonn 1955, S. 124. Berlin-Göttingen-Heidelberg: Springer 1956.

2. Bergquist, N.: The influence of pregnancy on diabetes. Acta endocr. 15, 166-181 (1954).

3. Bergström, S., and B. Borgström: Metabolism of lipids. Ann. Rev. Biochem. 25, 177-200 (1956).

4. Bletcher, S.J., C.F. Moldow, J. Scherrer, and M.G. GoldNER: A lipid mobilizing substance in the serum of pregnant women of probable placental origin. Metabolism 13, 583-586 (1964).

5. - J.G. O'Sullivan, and N. Freinket: Carbohydrate metabolism in pregnancy 5. The interrelations of glucose, insulin and free fatty acids in late pregnaney and post partum. New. Engl. J. Med. 271, 866-872 (1964).

6. Burt, R.L., N.H. LeAke, and R.P. Pulliam: Regulation of plasma NEFA in pregnancy and the puerperium. Amer. J. Obstet. Gynec. 17, 215-221 (1961).

7. - - W.N. DannenkuRG: Plasma nonesterified fatty acids in pregnancy. Amer. J. Obstet. Gynec. 84, 1081 -1090 (1962).

8. _ - - Plasma nonesterified fatty acids in pregnancy III Further observations on regulation of plasma NEFA-concentration by insulin and glucose. Amer. $\mathbf{J}$. Obstet. Gynec. 84, 1081-1090 (1962).

9. Cantre, G.F., B. Lerómu, and A. E. Renotn: Factors concerned with the regulation of fatty acid metabolism by adipose tissue. Amer. J. clin. Nutr. 8, $733-$ 739 (1960).

10. Camerint-Davalos, R.A., J.B. Cautfield, S.B. Rems, O. Lozano-Castaneda, S. NatdJIaN, and A. Marble: Proliminary observations on subjects with prodiabetes. Diabetes 12, 508-518 (1963).

11. Centonze, M., e P. SALviA DE: II lipoprotidogramma nella gravida normale a termine in corrispondenza del travaglio di parto. Quad. Clin. ostet., ginec. 11, 611$630(1956)$.

12. Conard, V., J.R.M. Franckson et P.A. Bastínte: Etude critique du triangle d'hyperglycémie intraveineux chez l'homme normal et détermination d'un "coefficient d'assimilation glucidique «. Arch. Int. Pharmacodyn. 93, 277-292 (1953).

13. Cramér, K., M. Aurmli, and S. Prehrson: Sorum lipids and lipoproteins in pregnancy. Clin. chim. Acta $10,470-476$ (1964).

14. Crostgnavi, P.G.: Determinazione immunologica del' insulina plasmatica in donne gravide. Ann. Ostet. Ginec. 82, 258-262 (1960).

15. Dole, V.P., A.T. James, J.P. Whep, M.A. Rizaok, and M.F. SturmanN: The fatty acid patterns of plasma lipids during alimentary lipemia. J. clin. Invest. 38, 1544-1554 (1959).

16. - Fractionation of plasma nonesterified fatty acid. Proc. Soc. exp. Biol. 93, 532-541 (1956).

17. ElerT, R.: Nebenniere und Schwangerschaft. Klin. Wschr. 19, 49-54 (1940).

18. FreiNKEL, N.: Effect of the conceptus on maternal metabolism during pregnancy. On the nature and treatment of diabetes. Excerpta medica foundation. $1965,679-691$. 
19. Gartand, P.B., and J.P. Randle: Regulation of glucose uptake by muscle. 10. Effects of diabetes, starvation, hypophysectomy and adrenalectomy and fatty acids, ketone bodies and pyruvate on glycerol output and concentrations of free fatty acids, long chain fatty acyl CoA, glycerol phosphate and citrate cycle intermediates in rat heart and diaphragm muscles. Biochem. J. 93, 678-687 (1964).

20. Gestaldi, A., e P.G. Crosignani: Modificazioni del'insulinomia in gravidanza dopo carico orale di glucosio. Ann. Ostet. Ginec. 81, 1171-1178 (1959).

21. GORDON, R.S., and A. ChERKES: Unesterified fatty acid in human blood plasma. J. clin. Invest. 35, 206$212(1956)$

22. Grady, H.J., and M.A. Lamar: Glucose determination by autonomic chemical analysis. - Clin. Chem. $5,542-550(1959)$.

23. Hausberger, F.X., S.W. Mristein, and R.J. RutMAN: The influence of insulin on glucose utilization in adipose and hepatic tissues in vitro. J. biol. Chem. $208,431-438$ (1954).

24. - Über die nervöse Regulation des Fettstoffwechsels. Klin. Wschr. 14, $77 \ldots 79$ (1935).

25. Hozt, J.P., A. Gommers, and J.J. Hoet: Clinical data on selected cases of prediabetes. In: OBERDISSE, K., and K. JAHNKF (Ed.): Diabetes mellitus, p. 529 . Stuttgart: Thieme-Verlag 1959.

26. - Praediabetische Schwangerschaften und fetale Pathologie. Verh. dtsch. Ges. inn. Med. 62, 643-653 (1956).

27. Hofrmann, W.S.: A Rapid Photoelectric Method for the Determination of Glucose in Blood and Urine. $J$. biol. Chem. 120, $51-55$ (1937).

28. HÜTER, K.A., and H. BLANK: Bedeutung, Erkennung und Behandlung prädiabetischer Stoffwechselsituationen in der Schwangerschaft. Geburtsh. und Frauen. heilk. 22, 612-630 (1962)

29. - W.H. ScHILLING, and H. Brank: Vergleichende Untersuchungen mit dem oralen und intravenösen Glukosetoleranztest in der Spätschwangerschaft. Z. Geburtsh. Gynäk. 164, 143-151 (1965).

30. JACKSON, W.P.U.: Effects of pregnancy on glucose tolerance. On the nature and treatment of diabetes, Excerpta Medica Foundation 1965, S. 718-721.

31. - Prediabetes a survey. J. Lab. clin. Med. 6, 127$157(1960)$.

32. Johansson, B.: Brown fat, a review. Metabolism 8, $221-240(1959)$

33. Jostmovich, J.B., and J.A. Mac LaReN: Presence in the human placenta and term serum of a highly lactogenic substance immunologically related to pituitary growth hormone. Endocrinology 71, 209-220 (1962).

34. KaplaN, S.L., and M.M. GRUMBACH: Studies of a human and similar placental hormone with growth hormone-like and prolactin-like activities. J. clin. Endocr. 24, 80-100 (1964).

35. Kersedy, E.P.: Metabolism of lipids. Ann. Rev. Biochem. 26, $119-142$ (1957).

36. Lambert, A.E., J.J. Howt, and E. EkKa: Plasma insulin levels during pregnancy in obesity and potential diabetes. Diabetologia 2, 260-264 (1966).

37. LochnER, W., u. M. NASSERr: Untersuchungen über den Herzstoffwechsel und Coronarduchblutung, insbesondere bei Dinitrophenolvergiftung. Pflügers Arch. ges. Physiol. 271, 405-419 (1960).

38. Murphy, R.: The hidden diabetic. Conn. State Med. J. 21, $306(1957)$.

39. Netson, G.H.: Serum nonesterified fatty acid levels in human pregnancy as determined by various titra. tion procedures. Amer. J. Obstet. Gynec. 92, $202 \ldots$ 206 (1965).

40. Randele, P.J., P.B. Gartand, N.C. Hales, and E.A. Newshoume: The glucose fatty acid cycle. Its role in insulin sensitivity and the metabolic disturbanees of diabetes mellitus. Lancet $1963 \mathrm{II}, 785-789$.

41. Roth, J., S.M. Glick, R.S. TALow, and S.A. Berson: Hypoglycemia: a potent stimulus to secretion of growth hormone. Science 140, 987-988 (1963).

42. Schildtag, W.H., K. Oberdisse, K.A. Hüter u. H. BLANK: Vergleichende Untersuchungen mit der oralen und intravenösen Glucosebelastung zur Erfassung einer verminderten Kohlenhydrattoleranz. Diabetologia 1, 187-194 (1965).

43. Scow, R.O., S.S. Chernick, and M.S. Brindey: Hyperlipemia and ketosis in the pregnant rat. Amer. J. Physiol. 206, 796-804 (1964).

44. SMITH, E.K., R.R. Alvarez DE, and J. Forsander: Serum protein, lipid and lipoprotein fraction in normal human pregnancy. Amer. J. Obstet. Gynec. 77, $326-$ $334(1959)$.

45. Spelcacy, W.N., and F.C. Goetz: Plasma insulin in normal late pregnancy. New Engl. J. Med. 268, 988991 (1963).

46. - - B.Z. GReerberg, and J. Elis: Plasma insulin in normal midpregnancy. Amer. J. Obstet. Gynec. 92, $11-15(1965)$.

47. WERTHEIMER, E., and B. Shaptro: The physiology of adipose tissue. Physiol. Rev. 28, 451-464 (1948).

48. Worm, M.: Diabetes und Gravidität. Dtsch. med. Wschr. 83, 802-811 (1958).

Dr. W.H. SCHULLING

Prof. Dr. K. JAHNKE

Dr. E. Derra

2. Med. Universitäts- und Poliklinik

4 Düsseldorf

Moorenstr. 5

Priv.-Doz. Dr. K.A. HüterR

Universitäts-Frauenklinik

4 Düsseldorf

Moorenstraße 5

Dr. L. HeRRERG

Diabetes-Forschungsinstitut an der Universität

4 Düsseldorf

Moorenstraße 5 